\title{
Synchronous Endometrial Malignant Mixed Mullerian Tumor and Ovarian High-Grade Serous Carcinoma: A Case Report and Review of Literature
}

\author{
${ }^{1}$ Department of Anatomic Pathology and Forensic Medicine, \\ Nnamdi Azikiwe University, Nnewi Campus, Anambra State, \\ Nigeria \\ ${ }^{2}$ Department of Anatomic Pathology and Forensic Medicine, \\ Nnamdi Azikiwe University, Nnewi Campus, Anambra State, \\ Nigeria \\ ${ }^{3}$ Department of Obstetrics and Gynaecology, Nnamdi Azikiwe \\ University Teaching Hospital, Nnewi, Anambra state, Nigeria

Felix E. Menkiti ${ }^{1}$ Ifeoma F. Ezejiofor ${ }^{2} \quad$ Michael E. Chiemeka ${ }^{2} \quad$ Chukwudi R. Osakwe $^{3}$

\author{
Address for correspondence Felix E. Menkiti, FMCPath, \\ Department of Anatomic Pathology and Forensic Medicine, \\ Nnamdi Azikiwe University, Nnewi Campus, Anambra State, Nigeria \\ (e-mail: menkiti_f@yahoo.com).
}

\begin{abstract}
Keywords

- endometrial malignant mixed Mullerian tumor

- serous ovarian carcinoma

- synchronous cancers

Synchronous cancers account for 0.7 to $1.8 \%$ of all gynecologic malignancies. Although synchronous endometrial and ovarian cancers are well reported in the literature, they are largely synchronous endometrioid cancers; no reported case of synchronous endometrial malignant mixed Mullerian tumor (MMMT) and ovarian serous carcinoma is seen. We therefore report a case of synchronous endometrial MMMT and ovarian serous carcinoma in a 60-year-old Nigerian female.
\end{abstract}

\section{Introduction}

Gynecological cancers account for $7.2 \%$ of cancers worldwide, with 1.6 and $2.1 \%$ occurring in the ovary and uterine corpus, respectively. ${ }^{1}$ Synchronous cancers account for 0.7 to $1.8 \%$ of all gynecologic malignancies, approximately $50 \%$ of which are accounted for by synchronous endometrial and ovarian cancers (SEOCs) ${ }^{2,3}$ SEOCs have been reported to account for approximately 3 to $10 \%$ and 3 to $5 \%$ of ovarian cancers and endometrial cancers, respectively. ${ }^{4,5}$ Matsuo et al in a retrospective study reported a changing trend in the pattern of SEOCs: a decreasing synchronous ovarian cancer among endometrial cancers and increasing synchronous endometrial cancer among ovarian cancer, and the peak age of incidence being late 40s. ${ }^{6}$ Although SEOC is well reported in literature, none has reported synchronous endometrial malignant mixed Mullerian tumor (MMMT) and ovarian high-grade serous carcinoma. However, there were reported cases of synchronous ovarian carcinoma in cases of MMMT of one ovary, broad ligament and mesentry ${ }^{7-10}$; and synchronous primary ovarian and uterine MMMT. ${ }^{11}$ We therefore report a case of MMMT of the endometrium occurring simultaneously with ovarian high-grade serous carcinoma in a 60 -year-old Nigerian female.

\section{Case Report}

A60-year-old postmenopausal female presented with a history of 5 months' recurrent vaginal bleeding and 1 month constipation with associated lower abdominal pain but no abdominal swelling. There was no family history of breast, ovarian, or colonic tumor. Physical examination of the abdomen revealed a bulky uterus with empty pouch of Douglas. Abdominopelvic sonogram revealed an endometrial mass with possible peritoneal seedlings, while chest radiogram was normal. Other investigations done, including serum electrolyte urea and creatinine, liver function test, full blood count, urinalysis, retroviral screening, hepatitis B virus surface antigen, and electrocardiography were all normal. Exploratory laparotomy was done, with subtotal hysterectomy and right salpingo-oophorectomy (and the sample sent to histopathology department for analysis), but constipation persisted necessitating further evaluation for possible primary or secondary colorectal tumor. received

August 30, 2019

accepted

October 23, 2019
DOI https://doi.org/

10.1055/s-0039-3401368

ISSN 2454-6798.
(C)2019 Spring Hope Cancer Foundation \& Young Oncologist Group of Asia
License terms

(ㅇ)(1) $\Theta \circledast$ 
Following evaluation, no lesion was noted in the breast and colorectum. Patient was discharged home 4 weeks later following good postoperation condition. Chemoirradiation was planned for the patient. About 8 weeks postsurgery, the constipation resolved and she had improved clinically. Patient could not receive the chemotherapy due to financial constraint and was subsequently lost to follow-up.

The collected surgical sample and peritoneal fluid were received in the histopathology department. The specimen consisted of subtotal hysterectomy and right salpingo-oophorectomy tissues, with ragged friable mass filling the endometrial cavity; the fallopian tube was unremarkable while the ovary which measured $7.0 \mathrm{~cm} \times 4.5 \mathrm{~cm} \times 3.0 \mathrm{~cm}$ has a smooth surface and a nodular cut surface (-Fig. 1). On histologic examination, the endometrium revealed a malignant mixed epithelial and mesenchymal neoplasm with high nuclear grade and frequent mitosis invading but not extending beyond the myometrium; features consistent with MMMT (-Figs. 2 and 3). The histologic sections of the right ovary showed invasive simple and complex arborizing papillae and glands lined by cells having high-grade nuclear features and moderate cytoplasm, with brisk mitosis and necrosis, consistent with high-grade serous carcinoma of the right ovary (-Fig. 4 ). The peritoneal aspirate cytology was negative for the tumor cells.

\section{Discussion}

SEOC is the simultaneous occurrence of both endometrial and ovarian cancers at the time of diagnosis. ${ }^{6}$ Although a rare instance, SEOC is the most frequent of the synchronous female genital tract cancers, ${ }^{2,7,12}$ which accounts for 0.7 to $1.8 \%$ of all gynecologic malignancies. ${ }^{2}$ SEOCs occur in younger women than those with either of primary ovarian or primary endometrial malignancies., ${ }^{5,6}$ A population-based retrospective analysis of SEOCs by Matsuo et al showed that among the endometrial cancer cohorts,

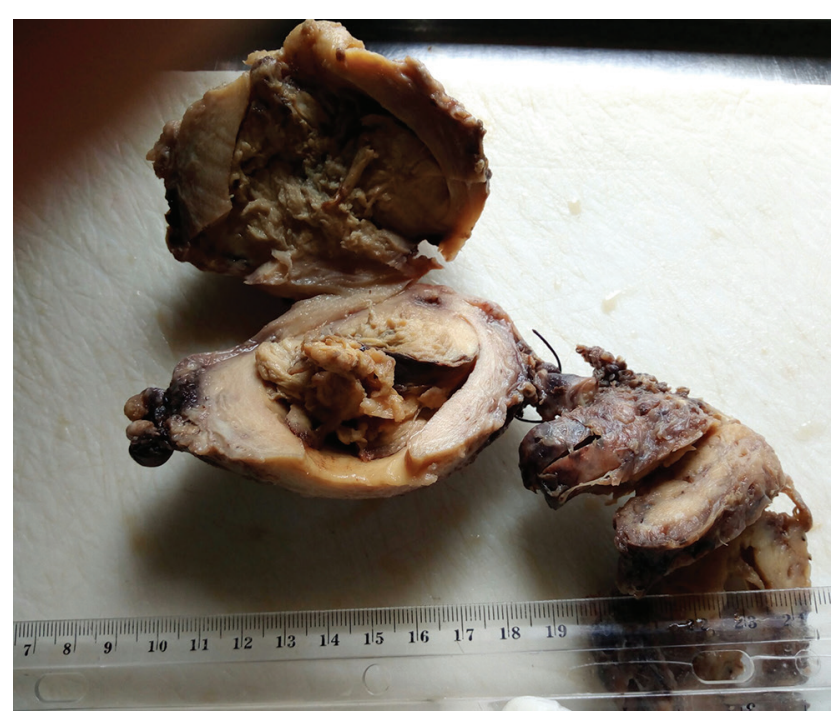

Fig. 1 A friable grayish white mass filling the endometrial cavity with a stump of unremarkable fallopian tube and attached right ovary showing circumscribed solid nodular lesion on cut section.

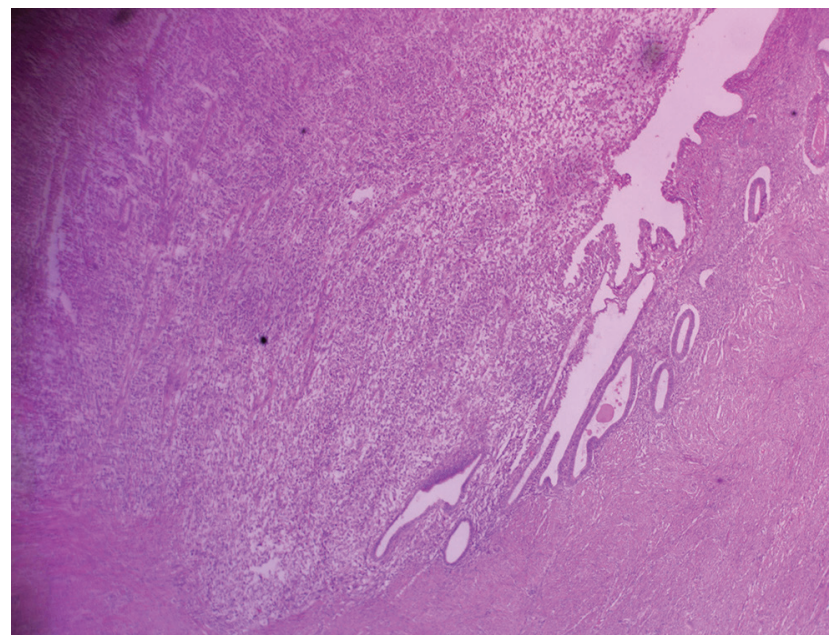

Fig. 2 Photomicrograph of malignant mixed Mullerian tumor of the endometrium invading but not extending beyond the myometrium $\times 40$.

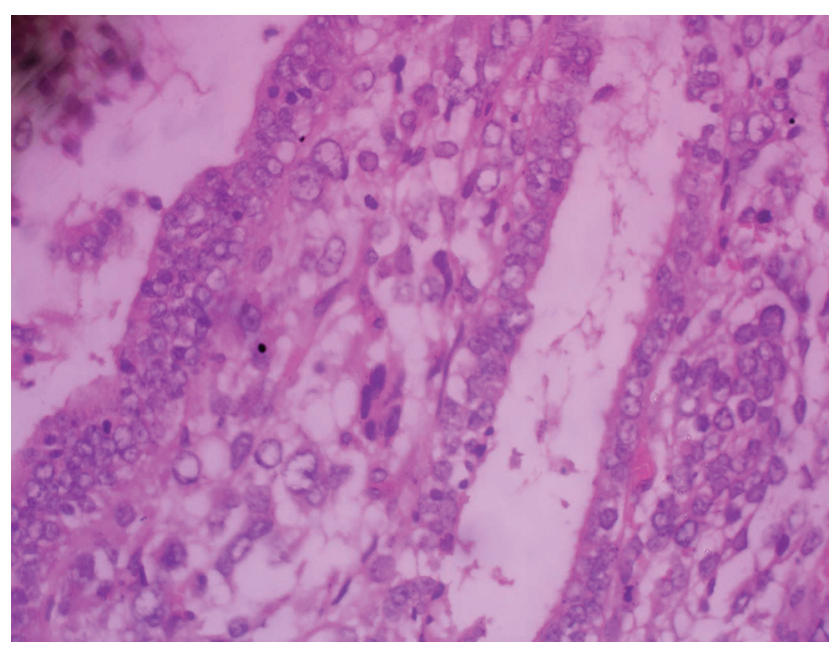

Fig. 3 (A and B) Higher magnification photomicrograph of malignant mixed Mullerian tumor of the endometrium showing high-grade nuclear features and abnormal mitosis (A, ×200; B, ×400).

synchronous primary endometrioid endometrial and ovarian tumors accounted for majority of the cases; while synchronous primary nonendometrioid ovarian and endometrial tumors accounted for only $16 \%$ of the cases, $21.8 \%$ accounted for the high-grade (grades 2 and 3 ) tumors. ${ }^{6}$ In their study, grade 3 synchronous primary nonendometrioid ovarian and endometrial carcinomas to which group the index case belongs, accounted for only $5 \%$ of the SEOCs. ${ }^{6}$

The study by Kobayashi et al showed that majority of SEOCs are sporadic cancers. ${ }^{13}$ However, several studies have been invested to unraveling the pathogenesis of SEOCs, which established a clonal relationship via gene sequencing. ${ }^{14}$ Several mutated genes have been found, some of which have syndromic association. Irving et al showed association between $\beta$-catenin mutations and SEOCs. ${ }^{15} \mathrm{~A}$ review by Dogan et al found microsatellite instability and subsequent mutations in mismatch repair genes compatible with hereditary nonpolyposis colorectal cancer (HNPCC) present in 40\% of women with SEOC and suggested a strong link between SEOC and HNPCC in young women. ${ }^{16}$ 


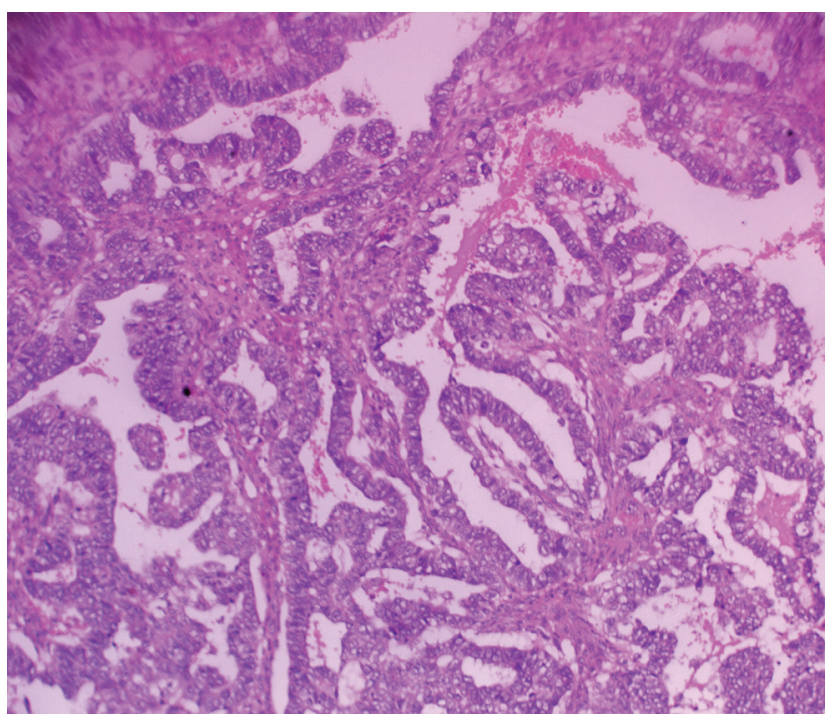

Fig. 4 Photomicrograph of the right ovary showing simple and complex arborizing papillae and glands lined by cells with high-grade nuclei and moderate cytoplasm $(\times 200)$.

Although the study by Brinkmann et al showed 53\% agreement between genetic and histologic diagnoses of synchronous tumors, they demonstrated that genetic characterization is of immense help in resolving many of the difficulties associated with distinguishing different tumor tissues by histopathology as being synchronous or metastatic. ${ }^{17}$ Hence, integrating molecular diagnosis in differentiating synchronous and metastatic tumors would be useful in the proper diagnosis of SEOC. ${ }^{18}$ This position is helpful if the tumors in both organs are similar. Diagnosing synchronous tumors in cases with dissimilar morphology as in the index case with endometrial MMMT and ovarian serous carcinoma is not problematic.

Distinguishing SEOC from metastatic cancers either of the ovary to the endometrium and vice versa is challenging, especially if the histologic type is same in the two cancer sites..$^{12}$ A set of guiding criteria developed for the histopathologic diagnosis of SEOC include, but are not limited to, the following ${ }^{19}$ : The two lesions must be histologically dissimilar as is seen in the index case with endometrial MMMT and ovarian serous carcinoma. Second, there should be no or only superficial myometrial invasion, no vascular space invasion by the endometrial tumor; this was the case in the index patient with the endometrial tumor still limited within the uterus (FIGO stage 1A) (-Fig. 2). Third, the synchronous ovarian tumor must be unilateral, located mainly in the parenchyma, and also show no vascular invasion or surface implants. The surface of the ovary of the index patient was smooth, and the tumor is located within the parenchyma, with no evidence of vascular space invasion.

Clinicopathologic correlation is also important in diagnosing synchronous tumors. Synchronous tumors usually occur within 1 year, else it could be metastatic or they are metachronic with earlier oncologic treatment as a probable carcinogen. ${ }^{2}$
Treatment of SEOCs is surgery with or without chemotherapy and radiotherapy depending on the grade and stage of the component cancers. ${ }^{2}$ The index patient had laparotomy with total hysterectomy and unilateral salpingo-oophorectomy, and was subsequently worked up for chemotherapy and radiotherapy.

Although several studies have reported a better prognosis for SEOCs compared with single occurring and metachronous tumors, the more informative population-based study by Matsuo et al, over a period of four decades, showed that survival of SEOC largely depends on the histologies of the two cancer sites. ${ }^{6}$ An international multicenter, case-control study also revealed that the prognosis of SEOCs is same with singly occurring endometrial or ovarian carcinoma when matched for age, FIGO stage, histology, and Eastern Cooperative Oncology Group performance status. ${ }^{20}$

\section{Conclusion}

SEOCs are rare group of tumors commonly presenting as synchronous primary endometrioid carcinomas of the ovary and endometrium. Synchronous nonendometrioid endometrial and ovarian cancer is even rarer, with no reported case of synchronous endometrial MMMT and ovarian serous carcinoma seen after extensive literature search. We have reported a rare case of synchronous endometrial MMMT and ovarian serous carcinoma.

\section{Conflict of Interest}

None declared.

\section{References}

1 Bray F, Ferlay J, Soerjomataram I, Siegel RL, Torre LA, Jemal A. Global cancer statistics 2018: GLOBOCAN estimates of incidence and mortality worldwide for 36 cancers in 185 countries. CA Cancer J Clin 2018;68(6):394-424

2 Dębska-Szmich S, Czernek U, Krakowska M, et al. Synchronous primary ovarian and endometrial cancers: a series of cases and a review of literature. Przegl Menopauz 2014;13(1):64-69

3 Ayhan A, Yalçin OT, Tuncer ZS, Gürgan T, Küçükali T. Synchronous primary malignancies of the female genital tract. Eur J Obstet Gynecol Reprod Biol 1992;45(1):63-66

4 Williams MG, Bandera EV, Demissie K, Rodríguez-Rodríguez L. Synchronous primary ovarian and endometrial cancers: a population-based assessment of survival. Obstet Gynecol 2009;113(4):783-789

5 AlHilli MM, Dowdy SC, Weaver AL, et al. Incidence and factors associated with synchronous ovarian and endometrial cancer: a population-based case-control study. Gynecol Oncol 2012;125(1):109-113

6 Matsuo K, Machida H, Blake EA, et al. Trends and outcomes of women with synchronous endometrial and ovarian cancer. Oncotarget 2018;9(47):28757-28771

7 Günakan E, Tohma YA, Haberal AN, Ayhan A. Bilateral synchronous ovarian tumours: an uncommon case and review of the literature. Przegl Menopauz 2018;17(2):97-100

8 Song MJ, Lee CW, Seo KJ, Kim JA, Park JS, Hur SY. A case of bilateral ovarian synchronous tumors (left ovarian serous papillary adenocarcinoma and right ovarian malignant mixed Müllerian tumor) Eur J Gynaecol Oncol 2011;32(2):234-236 
9 Ma CJ, Yang SF, Huang CC, et al. Malignant mixed Müllerian tumor of primary mesenteric origin associated with a synchronous ovarian cancer: case report and literature review. Eur J Gynaecol Oncol 2008;29(3):289-293

10 Arora P, Rao S, Khurana N, Talwar D, Tanwar R. Malignant mixed Mullerian tumor of broad ligament with synchronous ovarian and endometrial carcinoma: a rare association. J Cancer Res Ther 2011;7(1):88-91

11 Lee TY, Lee C, Choi WJ, Lee JY, Kim HY. Synchronous occurrence of primary malignant mixed Müllerian tumor in ovary and uterus. Obstet Gynecol Sci 2013;56(4):269-272

12 Makris GM, Manousopoulou G, Battista MJ, Salloum I, Chrelias G, Chrelias C. Synchronous endometrial and ovarian carcinoma: a case series. Case Rep Oncol 2017;10(2):732-736

13 Kobayashi Y, Nakamura K, Nomura H, et al. Clinicopathologic analysis with immunohistochemistry for DNA mismatch repair protein expression in synchronous primary endometrial and ovarian cancers. Int J Gynecol Cancer 2015;25(3):440-446

14 Schultheis AM, Ng CK, De Filippo MR, et al. Massively parallel sequencing-based clonality analysis of synchronous endometrioid endometrial and ovarian carcinomas. J Natl Cancer Inst 2016;108(6):djv427
15 Irving JA, Catasús L, Gallardo A, et al. Synchronous endometrioid carcinomas of the uterine corpus and ovary: alterations in the beta-catenin (CTNNB1) pathway are associated with independent primary tumors and favorable prognosis. Hum Pathol 2005;36(6):605-619

16 Dogan A, Schultheis B, Rezniczek GA, et al. Synchronous endometrial and ovarian cancer in young women: case report and review of the literature. Anticancer Res 2017;37(3):969-978

17 Brinkmann D, Ryan A, Ayhan A, et al. A molecular genetic and statistical approach for the diagnosis of dual-site cancers. J Natl Cancer Inst 2004;96(19):1441-1446

18 Anglesio MS, Wang YK, Maassen M, et al. Synchronous endometrial and ovarian carcinomas: evidence of clonality. J Natl Cancer Inst 2016;108(6):djv428

19 Lim YK, Padma R, Foo L, et al. Survival outcome of women with synchronous cancers of endometrium and ovary: a 10 year retrospective cohort study. J Gynecol Oncol 2011;22(4):239-243

20 Heitz F, Amant F, Fotopoulou C, et al. Synchronous ovarian and endometrial cancer-an international multicenter case-control study. Int J Gynecol Cancer 2014;24(1):54-60 